\title{
Topic Maps for Learning Design
}

\author{
Giovanni Adorni, Mauro Coccoli, Gianni Vercelli and Giuliano Vivanet \\ DIST - University of Genoa \\ Viale Causa 13, 16145 Genova, Italy \\ \{adorni, mauro.coccoli, gianni.vercelli, giuliano.vivanet\}@unige.it
}

\section{Summary}

Topic Maps (TM) are an ISO standard whose aim is describing knowledge structures and associating them with information resources. XML Topic Maps (XTM) is an XML-based encoding scheme to represent topic maps and similar knowledge structures. In this poster, TM and XTM are proposed as a knowledge representation system to be exploited in e-learning environments for a suited integration with semantic web technologies. They may be a useful tool to facilitate the design of learning contents and their delivery in different contexts.

The most fundamental elements in the TM paradigm are Topic, Association and Occurrence. According to ISO definition, a topic is a symbol used within a topic map to represent one subject, in order to allow statements to be made about the subject, that can be "anything whatsoever, regardless of whether it exists or has any other specific characteristics, about which anything whatsoever may be asserted by any means whatsoever". In substance a subject is anything about which the creator of a topic map chooses to discourse; for instance an object, an event, a place, a name, a concept, etc. An association represents a relationship between two or more topics. An occurrence is a representation of a relationship between a subject and an information resource.

Therefore, as it can be observed in the poster, two layers can be identified into this paradigm: a knowledge layer that represents topics and their relationships and an information layer that describes information resources. The existence of two different layers is one of the most interesting features of this model; in fact the same topic map could be used to represent different sets of information resources, or different topic maps could be used to represent the same resource repository.

Each topic can be featured by any number of names (and variants for each name); by any number of occurrences and by its association role, that is a representation of the involvement of a subject in a relationship represented by an association. All these 
features are statements and have a scope that represents the context within which a statement is valid. Using scopes it is possible to remove ambiguity about topics; to provide different points of view on the same topic (based on users' profile) and/or to modify each statement depending on users' language, etc. Topics, topic names, occurrences, associations and associations' roles require a type element (becoming instances of classes). These classes are also topics, which might, again, be instances of other classes. Therefore, to solve ambiguity issues, each subject, represented by a topic, is identified by a subject identifier (usually a URI, similarly to RDF). This unambiguous identification of subjects is also used in TM to merge topics that, through these identifiers, are known to have the same subject. This feature could be used in order to share the knowledge and to solve redundancy issues.

The proposed scenario is considering the use of the TM paradigm as a tool for the design of educational paths and contents. This standard could be profitably considered as a mean for describing the table of contents of a course as well as the outline of a lesson according to the logical structure of the course itself. In addition, semantic information is stored within the TM in a well formed form and in a standard language, thus it can be easily exported over the Internet and many systems can re-use and interoperate with the XTM representation of the topic map. Moreover, the layered structure also enables authors to define different maps based on a common repository of resources so that personalized learning paths can be defined while the contents at the occurrence level remain the same and different educational strategies can be implemented.

In the context of education, TM can be used as a means to express knowledge and to organize and retrieve information in a more efficient and meaningful way. According to Koper "an important question related to the educational semantic web, is how to represent a course in a formal, semantic way so that it can be interpreted and manipulated by computers as well as humans". The semantic representation of learning courses opens the possibility to solve parts of some problems such as the interoperability of educational contents; the development of flexible, problem-based, non-linear and personalized web-based courses; the building and sharing catalogues of learning and teaching patterns; the adaptation to learners' profile and the semantic research capabilities. 